\title{
Infections caused by Stenotrophomonas maltophilia in recipients of hematopoietic stem cell transplantation
}

\author{
Khalid Ahmed Al-Anazi ${ }^{1}{ }^{*}$ and Asma M. Al-Jasser ${ }^{2}$ \\ Section of Adult Hematology and Oncology, Department of Medicine, College of Medicine, King Khalid University Hospital, King Saud University, \\ Riyadh, Saudi Arabia \\ ${ }^{2}$ Central Laboratory, Ministry of Health, Riyadh, Saudi Arabia
}

\section{Edited by:}

Gautam Borthakur, MD Anderson

Cancer Center, USA

\section{Reviewed by:}

Paul Armistead, University of North

Carolina, USA

Vikram Mathews, Christian Medical

College, India

Gautam Borthakur, MD Anderson

Cancer Center, USA

*Correspondence:

Khalid Ahmed Al-Anazi, Section of Adult Hematology and Oncology,

Department of Medicine, College of Medicine, King Khalid University

Hospital, King Saud University, P.O.

Box: 2925, Riyadh 11461, Saudi Arabia e-mail: kaa_alanazi@yahoo.com
Stenotrophomonas maltophilia (S. maltophilia) is a globally emerging Gram-negative bacillus that is widely spread in environment and hospital equipment. Recently, the incidence of infections caused by this organism has increased, particularly in patients with hematological malignancy and in recipients of hematopoietic stem cell transplantation (HSCT) having neutropenia, mucositis, diarrhea, central venous catheters or graft versus host disease and receiving intensive cytotoxic chemotherapy, immunosuppressive therapy, or broad-spectrum antibiotics. The spectrum of infections in HSCT recipients includes pneumonia, urinary tract and surgical site infection, peritonitis, bacteremia, septic shock, and infection of indwelling medical devices. The organism exhibits intrinsic resistance to many classes of antibiotics including carbapenems, aminoglycosides, most of the thirdgeneration cephalosporins, and other $\beta$-lactams. Despite the increasingly reported drug resistance, trimethoprim-sulfamethoxazole is still the drug of choice. However, the organism is still susceptible to ticarcillin-clavulanic acid, tigecycline, fluoroquinolones, polymyxin$B$, and rifampicin. Genetic factors play a significant role not only in evolution of drug resistance but also in virulence of the organism. The outcome of patients having S. maltophilia infections can be improved by: using various combinations of novel therapeutic agents and aerosolized aminoglycosides or colistin, prompt administration of in vitro active antibiotics, removal of possible sources of infection such as infected indwelling intravascular catheters, and application of strict infection control measures.

Keywords: Stenotrophomonas maltophilia, bacteremia, neutropenia, hematopoietic stem cell transplantation, drug resistance

\section{INTRODUCTION}

Stenotrophomonas maltophilia (S. maltophilia) is a free living, motile, aerobic, oxidase negative, glucose non-fermentative Gramnegative bacillus (GNB). It is frequently isolated from water, soil, animals, plants, and hospital equipment (1-5). S. maltophilia is the only species of the genus Stenotrophomonas known to infect humans. The organism was first isolated from pleural fluid by Edwards in 1943 and it was called Bacterium brookeri $(1,3)$. Hugh and Ryschenkow reclassified Bacterium brookeri as Pseudomonas maltophilia in 1961. Twenty years later, Swings et al. proposed reclassification of $P$. maltophilia as Xanthomonas maltophilia. In the year 1993, the genus Stenotrophomonas was proposed by Palleroni and Bradbury, so the organism was finally named as S. maltophilia (1). Risk factors for S. maltophilia infections in the general population are very variable and they are listed in Table 1 (1-14).

\section{INFECTIONS CAUSED BY S. MALTOPHILIA}

S. maltophilia causes various infectious complications in immunocompromised individuals and these include bacteremia, endocarditis, respiratory tract infections, meningitis, urinary tract infections, skin and soft tissue infections, mastoiditis, bone and joint infections, peritonitis, typhlitis and biliary sepsis, wound infections, and central venous catheter (CVC)-related infections $(1-3,5-7,11,15-17)$. Occasionally, distinguishing between colonization and infection can be problematic (1-3). S. maltophilia infections can be complicated by septic shock, respiratory failure, pulmonary hemorrhage, metastatic cellulitis, tissue necrosis that may be extensive, septic thrombophlebitis, disseminated infection, and death $(5-8,11)$.

\section{S. MALTOPHILIA INFECTIONS IN RECIPIENTS OF HSCT}

Recipients of various forms of hematopoietic stem cell transplantation (HSCT) are at risk of a wide range of infectious complications due to their severely suppressed immunity. Infections caused by $S$. maltophilia cause significant morbidity and mortality in this group of patients $(2,5,7,9,10,13,14)$. Peculiar risk factors predispose recipients of HSCT to $S$. maltophilia infections and these are included in Table $2(2,5,7,9,10$, $13,14)$. The most commonly encountered infectious complications related to S. maltophilia are pneumonia, bacteremia, and CVC-related infections. S. maltophilia may coexist with other infections caused by bacterial, viral, fungal, and protozoal agents $(5,6)$.

S. maltophilia infections have been described in various forms of HSCT: autologous transplant and allogeneic, sibling related, 
Table 1 | Risk factors for $\boldsymbol{S}$. maltophilia infections in the general population.

(1) Malignancy, particularly hematological malignancy

(2) Human immunodeficiency virus (HIV)

(3) Cystic fibrosis

(4) Intravenous drug abuse

(5) Surgical and accidental trauma

(6) Prolonged hospitalization

(7) Admission to ICU and mechanical ventilation

(8) Indwelling vascular catheters and urinary catheters

(9) Corticosteroids and immunosuppressive therapy

(10) Prior treatment with broad-spectrum antibiotics

(11) Gastrointestinal tract colonization and mucositis

(12) Hematopoietic stem cell transplantation (HSCT)

(13) Travel to hospital by air

ICU, intensive care unit.

Table 2 | Risk factors for S. maltophilia infections in HSCT recipients.

(1) Underlying disease being a hematological malignancy:

leukemia, lymphoma, or multiple myeloma

(2) Cytotoxic chemotherapy

(3) Radiotherapy

(4) Neutropenia and bone marrow aplasia

(5) Graft versus host disease (GVHD)

(6) Immunosuppressive therapies:

corticosteroids and cyclosporine-A

(7) Monoclonal antibodies

(8) Indwelling vascular catheters and urinary catheters

(9) Prior treatment with broad-spectrum antibiotics

(10) Prolonged hospitalization

(11) Admission to ICU and mechanical ventilation

(12) Gastrointestinal tract colonization and diarrhea

(13) Severe mucositis

$I C U$, intensive care unit.

matched unrelated donor and umbilical cord blood, HSCT (5, 7-9, 13, 14).

\section{CVC INFECTIONS CAUSED BY S. MALTOPHILIA}

Central venous catheter-related infections are the most frequent cause of hospital-acquired bacteremia in critically ill patients. Over the past two decades, the incidence of CVC-related bacteremia due to GNB has increased. Among non-fermentative GNB, S. maltophilia is the most frequently isolated pathogen (18). In patients with cancer, hematological malignancy (HM), and in recipients of HSCT acquiring bacteremia, CVC-related infection should be considered seriously as S. maltophilia infections are commonly associated with the presence of CVCs in these immunocompromised hosts $(13,19,20)$. S. maltophilia CVC-related infections have also been reported in HSCT recipients. These infections cause bacteremia that may be recurrent, may occur as part of polymicrobial infection, and carry high mortality rates $(2,5,13,14)$.

Indications for removal of CVCs include: (1) bloodstream infection (BSI) due to GNB particularly multidrug-resistant (MDR) isolates, (2) critically ill patients such as high risk immunocompromised hosts including patients with HM and recipients of HSCT, and (3) presence of complications such as: severe sepsis and septic shock, tunnel infection, suppurative thrombophlebitis, and infective endocarditis (21). Administration of appropriate antibiotic therapy, immediate removal of infected catheters, and implementation of strict hand hygiene for health-care personnel are crucial in the management of CVC-associated infections and are associated with good prognosis $(2,5,13,14,19,20)$.

In patients with documented CVC-related GNB infections, removal of CVC should be performed within 48-72 h $(20,22)$. However, some studies have reported that, even in patients with long-term CVC having infections caused by GNB, catheter salvage can be achieved in $70 \%$ of cases by antimicrobial therapy and decontamination of the catheter lock with an anti-infective lock solution (23). Novel securement devices and antibiotic lock solutions have also been shown to reduce the risk of intravascular device-related BSIs in prospective randomized trials (24). Other studies have shown that, despite the use of appropriate antibiotics, keeping an infected catheter in situ causes not only recurrence in infection by the same GNB or other micro-organisms but also death $(20,22)$. Recurrence of CVC-related infections has been reported after long latency period reaching 200 days (20).

\section{PNEUMONIA CAUSED BY S. MALTOPHILIA}

In hospitalized HSCT recipients, the respiratory tract is the most common site of isolation of the organism $(2,3)$. In recipients of HSCT, the risk factors for hospital-acquired pneumonia due to $S$. maltophilia include: (1) immunocompromised health status, (2) indwelling CVCs, (3) mechanical ventilation, (4) exposure to and duration of broad-spectrum antibiotic therapy, (5) prolonged hospitalization, (6) admission to an intensive care unit (ICU), and (7) presence of underlying lung disease such as chronic obstructive airway disease $(25,26)$. Treatment of ventilator-associated pneumonia (VAP) caused by S. maltophilia includes: (1) high dose trimethoprim-sulfamethoxazole (TMP-SMZ), which is still considered the drug of choice based on its excellent in vitro activity, and (2) an alternative antimicrobial therapy in patients having drug resistance, allergy, or adverse effects related to TMP-SMZ that includes a new fluoroquinolone or a combination of doxycycline and aerosolized colistin $(27,28)$. Despite the recent improvement in the short-term prognosis of HSCT recipients transferred to ICU, S. maltophilia-associated VAP and bacteremia still carry significant morbidity and mortality $(28,29)$.

In patients with HM and in recipients of HSCT having severe lung infection caused by $S$. maltophilia, life-threatening pulmonary alveolar hemorrhage (PAH) causing acute respiratory failure has been reported $(7,8,30-32)$. The risk factors for PAH in these patients include prolonged cytopenias and high C-reactive protein levels at the onset of BSI or pneumonia $(7,8)$. However, 
the mechanism of $S$. maltophilia-induced PAH remains unclear $(7,8)$. Despite the expected dismal outcome, prompt institution of TMP-SMZ in combination with other antimicrobials, provision of advanced respiratory care, and adequate blood products including granulocyte transfusions, if needed, may be life-saving in these immunocompromised individuals $(7,8,30-32)$. In a study that included 223 recipients of HSCT who had diffuse pulmonary infiltration with respiratory compromise: $39 \%$ of patients had $\mathrm{PAH}$ and $43 \%$ of patients had an organism cultured from BAL. Patient who had PAH and an organism isolated from BAL had the worst outcome and isolation of a microbial organism from BAL was a strong predictor of poor prognosis (31).

\section{BACTEREMIA CAUSED BY S. MALTOPHILIA}

S. maltophilia bacteremia is hospital-acquired in $76 \%$ of patients and has been reported in up to $24 \%$ of recipients of allogeneic HSCT $(9,18)$. The risk factors for S. maltophilia bacteremia are included in Table 3 (5, 6, 9, 12, 14, 18, 19, 33-36). The sources of infection in patients having $S$. maltophilia bacteremia include: primary bacteremia with no known source, CVC-associated infections, respiratory tract infections such as pneumonia in mechanically ventilated patients, gastrointestinal tract (GIT) infections, and faulty replacement of hand washing soap $(5,6,9,10,14)$. Outbreaks of S. maltophilia bacteremia have been described in recipients of allogeneic HSCT having neutropenia and mechanical ventilation $(9,10)$. These outbreaks were attributed to: (1) hospital equipments acting as reservoirs for $S$. maltophilia, (2) prevalence of GIT colonization with the organism in patients who develop invasive infections, (3) selective pressure of antimicrobial therapy, and (4) faulty replacement of hand washing soap $(9,10)$.

The overall mortality related to $S$. maltophilia bacteremia may reach $33 \%(5,18)$. Studies have also shown that 14 -day and 30-day mortality rates in patients having S. maltophilia BSIs are 24 and $35 \%$, respectively $(6,37)$. The 30-day mortality rate related to $S$. maltophilia bacteremia is higher and has worse

\section{Table 3 | Risk factors for S. maltophilia bacteremia.}

(1) CVC infection

(2) Prolonged hospitalization

(3) Admission to ICU and mechanical ventilation

(4) Prior treatment with broad-spectrum antibiotics

(5) Severe neutropenia

(6) Corticosteroid treatment

(7) Underlying disease being a hematological malignancy

(8) Aggressive cytotoxic chemotherapy

(9) Immunosuppressive therapy

(10) Major trauma or recent surgical intervention

(11) Severe mucositis

(12) Total parenteral nutrition

$I C U$, intensive care unit; CVC, central venous catheter. prognosis compared with bacteremia related to other glucose nonfermenting GNB such as Pseudomonas aeruginosa and Acinetobacter species (37). Factors independently associated with mortality in patients having $S$. maltophilia bacteremia include: (1) profound neutropenia, (2) thrombocytopenia, (3) septic shock or hypotension at the onset of bacteremia, (4) inappropriate antimicrobial therapy, and (5) having high sepsis-related organ failure assessment index $(6,14,18)$. In patients having $S$. maltophilia BSIs, good prognosis is associated with early administration of appropriate antimicrobial therapy, removal of infected CVCs, recovery of bone marrow function, and taking enough preventive and isolation measures $(5,14,35,36)$.

\section{ANTIMICROBIAL SUSCEPTIBILITY AND THERAPEUTIC OPTIONS}

S. maltophilia has been shown to be susceptible to the following antibiotics: (1) TMP-SMZ, (2) ticarcillin-clavulanate, (3) piperacillin-tazobactam, (4) cephalosporins such as ceftazidime, (5) aminoglycosides such as streptomycin and amikacin, (6) tetracyclines such as minocycline, doxycycline, and tigecycline, (7) fluoroquinolones such as ciprofloxacin, levofloxacin, moxifloxacin, gatifloxacin, and trovafloxacin, (8) polymyxins such as colistin sulfate, and (9) other antibiotics such as rifampicin, chloramphenicol, salbactam-cefoperazone, moxalactam as well as silver sulfadiazine $(1,3,5-7,10,11,14,15,38-48)$.

The organism has been reported to be resistant to the following antimicrobials: (1) carbapenems such as imipenem and meropenem, (2) amoxicillin-clavulanic acid, (3) aminoglycosides such as tobramycin and gentamicin, (4) most of the cephalosporins such as cefpirome and cefsulodin, (5) quinolones and most of the anti-pseudomonal penicillins. Also, some strains have been reported to be resistant to tetracyclines, ciprofloxacin, chloramphenicol $(1,5,10,11,16,39,49-51)$.

In a large survey (CANWARD 2007-11 Study) performed in Canada between 2007 and 2001 that included 22,746 clinical isolates from hospitals in 8 districts: $S$. maltophilia accounted for $1.4 \%$ of all isolates and the organism ranked number 16 among the 20 most common pathogens isolated from Canadian hospitals (42). Also, in a 6-month prospective multicenter study performed in 9 teaching hospitals in France that included 158 patients, $S$. maltophilia was the most common non-fermentative GNB (39\%) isolated from hospitalized patients (52). In the former study, tigecycline displayed good in vitro activity against MDR isolates of S. maltophilia and in the latter study the high incidence of MDR among S. maltophilia isolates rendered empirical antimicrobial therapy inappropriate $(42,52)$.

Various studies have shown synergistic activity when the following combinations are used: TMP-SMZ and ticarcillinclavulanate, TMP-SMZ and ceftazidime, ticarcillin-clavulanate and levofloxacin, ticarcillin-clavulanate and aztreonam as well as ceftazidime and ciprofloxacin (43-45). Only one study showed that TMP-SMZ and fluoroquinolones are equally effective once used as monotherapies in treating $S$. maltophilia-related infections (53). MDR isolates of S. maltophilia have shown excellent responses to combinations of new fluoroquinolones and tetracycline derivatives (46). Future therapies that have the potential to slow the development of drug resistance include phage therapy, 
nanoemulsions, use of antisense RNA to target genes involved in virulence, and use of adjuvants that restore antibiotic efficacy (43).

Studies on in vitro susceptibility of $S$. maltophilia performed in several countries have shown variable results $(42,47,48,52-$ 56). A Greek 6-year survey showed that in vitro susceptibility of $S$. maltophilia to TMP-SMZ, colistin, netlimicin, and ciprofloxacin were 85.3, 91.2, 85.3, and 82.4\%, respectively (47). A Spanish survey showed: (1) S. maltophilia was found to be highly resistant to several antibiotics, and (2) TMP-SMZ and ticarcillin-clavulanate were the only antimicrobials that exhibited good in vitro activity against S. maltophilia (48). One Korean study showed that susceptibility rates of $S$. maltophilia to TMP-SMZ, minocycline, and levofloxacin were 96, 99, and 64\% respectively (54). Another Korean survey showed: (1) the degree of antimicrobial resistance, even to TMP-SMZ, varied considerably between isolates, and (2) accurate identification was essential for appropriate selection of treatment options and alternative therapies in case of TMPSMZ resistance (55). Finally, a study performed in Saudi Arabia showed that in vitro resistance rates of $S$. maltophilia to various antibiotics were as follows: imipenem $100 \%$, gentamicin $87.4 \%$, aztreonam $86 \%$, ciprofloxacin $77 \%$, piperacillin-tazobactam $61 \%$, ceftazidime 57\%, and TMP-SMZ 9.5\% (56).

\section{DRUG RESISTANCE IN S. MALTOPHILIA}

Unfortunately, the organism is resistant to several antimicrobials and there is a growing concern about the magnitude of drug resistance displayed by S. maltophilia (2-4, 17, 40, 51, 57-63). There are several mechanisms of drug resistance and these are listed in Table 4. Genetic determinants of drug resistance in S. maltophilia include class 1 integrons carrying sul 1 gene and insertion sequence

Table 4 | Mechanisms of $S$. maltophilia drug resistance.

\begin{tabular}{|c|c|}
\hline Mechanism & Examples \\
\hline $\begin{array}{l}\text { (1) Modification of outer membrane } \\
\text { proteins }\end{array}$ & $\begin{array}{l}\text { - Protein expression } \\
\text { - Reduction in permeability }\end{array}$ \\
\hline $\begin{array}{l}\text { (2) Expression of chromosomally } \\
\text { encoded multidrug efflux pumps }\end{array}$ & $\begin{array}{l}\text { - Sme ABC } \\
\text { - Sme DEF } \\
\text { - Cadmium efflux determinants: } \\
\text { Cad A and Cad C } \\
\text { - Resistance-nodulation cell } \\
\text { division (RND) efflux pump. }\end{array}$ \\
\hline (3) Enzyamatic mechanisms & $\begin{array}{l}\text { - Expression of chromosomal or } \\
\text { plasmid encoded } \beta \text {-lactamases } \\
\text { such as } L_{1} \text { and } L_{2} \beta \text {-lactamases } \\
\text { - Aminoglycoside acetylcholine } \\
\text { modifying enzyme a }\left(6^{\prime}\right) \text { Iz gene. } \\
\text { - Inactivation of macrolide } \\
\text { phosphotransferase. }\end{array}$ \\
\hline (4) Target site alterations & $\begin{array}{l}\text { - Phosphoglucomutase gene } \\
\text { mutations on liposaccharide. } \\
\text { - Mutations of bacterial } \\
\text { topoisomerase and gyrase } \\
\text { genes. }\end{array}$ \\
\hline
\end{tabular}

common region (ISCR) elements carrying sul 2 gene (Table 5) (2$4,17,40,51,57-63)$. The mechanisms of drug resistance vary from one antimicrobial to another and at times each antibiotic may have different enzymes or genes involved in the evolution of its drug resistance. For example: class 1 integrons $\left(\mathrm{sul}_{1}\right)$ and ISCR elements (sul 2) are involved in the resistance to TMP-SMZ, sme DEF, and smQnr are involved in quinolone resistance and three different mechanisms, aac $\left(6^{\prime}\right)$ Iz acetyl transferase modifying enzyme, efflux pumps, and outer membrane modifications, are involved in drug resistance to aminoglycosides (Tables 4 and 5) $(51,57,58,62)$.

Several molecular mechanisms contribute to MDR and they include plasmids, integrons, and transposons (2). Resistance of $S$. maltophilia is mediated by a relatively impermeable cell membrane, the production of $\beta$-lactamases $\left(\mathrm{L}_{1}\right.$ and $\left.\mathrm{L}_{2}\right)$, and an efficient resistance nodulation cell division (RND) efflux pump Sme DEF able to extrude fluoroquinolones, chloramphenicol, and tetracycline (64). Biofilm formation leads to colonization of mucosal surfaces, wounds, fluids, catheters, and implants and this contributes to persistence of the organism $(2,65,66)$. Persistence of $S$. maltophilia, virulence of the bacterium, and inappropriate use of broad-spectrum antibiotics predispose to drug resistance (2).

\section{Table 5 | Genetic determinants of drug resistance}

\section{Genetic determinant \\ Antibiotics involved in drug resistance}

Sul 1 and Sul 2

Trimethoprim-sulfamethoxazole

Sm Onr R

ISCR

Quinolones, tetracyclines.

Sme ABC

Trimethoprim-sulfamethoxazole, quinolones, aminoglycosides, $\beta$-lactams

Sme DEF

Sme IJK

Quinolones such as ciprofloxacin, aminologycosides, $\beta$-lactams

- Quinolones such as ciprofloxacin and ofloxacin

- Macrolides

- Chloramphenicol

- Tetracycline

- Novobiocin

- Quinolones such as ciprofloxacin and levofloxacin

- Aminoglycosides

- Tetracyclines

Sme OP and Tol C sm

- Aminoglycosides such as gentamicin and amikacin

- Trimethoprim-sulfamethoxazole

- Nalidixic acid

- Chloramphenicol

- Erythromycin and other macrolides

- Doxycycline

- Leucomycin

- Chemicals such as tetrachlorosalicylanilide.

Sme Z

- Aminoglycosides 
Studies have shown that genetic factors play a major role in drug resistance $(2,67)$. The wide range of antimicrobial drug resistance genes and mobile genetic elements found suggests that S. maltophilia can act as a reservoir of antimicrobial drug resistance determinants in a clinical environment, which is an issue of considerable concern (67). The genome sequence of S. maltophilia reveals its capacity for environmental adaptation that presumably contributes to its persistence in vivo (67). The genome sequencing of S. maltophilia isolates K 279a and AU 12-09 revealed huge number of genes coding for MDR to several antimicrobials $(67,68)$. Although it is not a highly virulent organism, the large number of genes involved, the MDR phenotype, and its ability to attach to mucosal surfaces and surfaces of hospital equipment make the organism more persistent and more difficult to eradicate (67).

S. maltophilia has high intrinsic resistance to a variety of structurally unrelated antimicrobials including aminoglycosides, quinolones, and $\beta$-lactams. Multidrug efflux transport systems are an important mechanism for bacteria to combat antimicrobial agents. Among efflux transport systems, the RND-type multidrug efflux systems play a critical role in MDR, particularly in GNB (69). Two efflux systems have been identified in S. maltophilia, Sme ABC and Sme DEF. Genome sequence analysis has revealed that S. maltophilia K279a encodes many putative RND efflux systems including Sme ABC, Sme DEF, Sme GH, Sme IJK, Sme MN, Sme OP, Sme VWX, and Sme YZ. Sme $\mathrm{U}_{1}-\mathrm{V}-\mathrm{W}-\mathrm{U}_{2}-\mathrm{Y}$, which is a novel RND-type efflux pump operon, composed of five genes, has been characterized (69). The SmQnr protein encoded by the chromosome qur determinant of $S$. maltophilia has been shown to contribute to the intrinsic quinolone resistance of S. maltophilia. Inactivation of SmqnrR contributes to an acquired increase in quinolone and tetracycline minimum inhibitory concentrations (MICs) for S. maltophilia (Table 5) (70).

A five-gene cluster (tolCsm, Sme RO, Sme D, Sme P, and pcm) of S. maltophilia was characterized (71). The pcm-tolCsm makes a significant contribution to multidrug resistance of $S$. maltophilia (72). Deletion of tolCsm increases susceptibility of S. maltophilia $\mathrm{KJ}_{2}$ to: (1) several antimicrobial agents including aminoglycosides, macrolides, doxycycline, nalidixic acid, chloramphenicol, and TMP-SMZ, and (2) chemical compounds including carbonylcyanide 3-chlorophenyl hydrazine, tetrachlorosalicylanilide, fusidic acid, paraquat, crystal violet, menadine, and plumbagin (72). The sul 1 and sul 2 genes lead to a high rate of TMP-SMZ resistance (Table 5) (73). ISCR elements are associated with a variety of antibiotic resistance genes. At least 13 ISCRs have been identified and they are associated with resistance to TMP-SMZ, quinolones, aminoglycosides, and $\beta$-lactams (74).

The increasing number of $S$. maltophilia isolates seen in ICUs, their resistance to mainstay antibiotics, their genetically diverse nature and possible cross-transmission within hospitals strongly underscore the need for continuous surveillance for S. maltophilia in hospital settings (75). Environmental isolates of S. maltophilia have been found to have lower overall mutation frequencies compared with clinical isolates (76). Trichloroethylene (TCE) is one of the major ground water pollutants throughout the world (77). S. maltophilia PM102 is capable of growth on TCE as the sole carbon source and has the capacity to degrade TCE efficiently $(77,78) . S$. maltophilia PM102 has been identified by $16 \mathrm{~S}$ rDNA sequencing and has been characterized by Fourier transform infrared spectroscopy (FT-IR) $(78,79)$. Triclosan is one of the most widely used biocides. Binding of triclosan to Sme T induces antibiotic resistance in S. maltophilia. Triclosan is a good inducer of the expression of MDR efflux pump Sme DEF and exposure of $S$. maltophilia to triclosan selects for antibiotic resistance (80).

\section{NEW DIAGNOSTIC METHODS}

As infections caused by $S$. maltophilia are associated with considerable morbidity and mortality and as the organism has the potential to acquire resistance to many classes of antimicrobials, it is essential to make diagnosis and identification of the organism as early as possible in order to institute appropriate antibiotic therapy promptly. This can be achieved by utilizing the new or advanced laboratory and radiological diagnostic tools (81).

\section{MOLECULAR DIAGNOSTICS IN S. MALTOPHILIA INFECTIONS}

Detection of $S$. maltophilia in blood cultures can be achieved more rapidly by combining flow cytometry and fluorescence in situ hybridization compared to standard detection methods (81). Both conventional and real-time polymerase chain reaction (PCR) are sensitive methods for diagnosing GNB infections including those caused by $S$. maltophilia in cancer patients having febrile neutropenia. Although PCR is more costly than routine blood cultures, its use in patients with HM having febrile neutropenia can be justified as studies have shown that the use of PCR under such circumstances has improved the outcome and has reduced the mortality related to these serious infections (81-83).

Early identification of pathogens and antimicrobial resistance in BSIs decreases morbidity and mortality in patients with HM and in recipients of HSCT (83-85). Molecular techniques, such as PCR, ribosomal RNA, and nucleic acid amplification, can augment cultural methods in the diagnosis of causal agents of bacteremia in these patients so that appropriate antimicrobial therapies can be commenced particularly in culture-negative infection episodes $(83,85)$. Although blood cultures remain the gold standard for detecting BSIs, real-time PCR is a valuable complementary tool in the management of BSIs in recipients of HSCT, thus allowing early identification of pathogens and antimicrobial resistance genes (84).

The LightCycler SeptiFast assay, a multiple blood PCR technique, when combined with blood cultures provides clinically relevant information for the diagnosis of blood culture-negative febrile neutropenia in patients with persistent fever despite antibacterial therapy or when a non-responding bacterial infection or an invasive fungal infection is suspected $(86,87)$. Results of the SeptiFast assay may lead to a more targeted antimicrobial treatment early after the onset of fever (88).

\section{DIAGNOSTICS IN PULMONARY COMPLICATIONS OF S. MALTOPHILIA}

In patients with febrile neutropenia, computed axial tomography (CAT) scan of the thorax is more sensitive than chest X-ray (CXR) in detecting pulmonary infections (89). Positron emission tomography scans are also valuable in defining the extent of infection and guiding management in immunocompromised individuals having febrile neutropenia (90).

In febrile neutropenic patients, having a normal CXR does not rule out pneumonia. High resolution CAT (HR-CAT) scans can 
detect pneumonia 5 days earlier than $\operatorname{CXR}(91,92)$. Therefore, patients with febrile neutropenia with no evidence of pneumonia on CXR should have HR-CAT scans $(91,92)$. The most common HR-CAT scan findings in recipients of HSCT having pneumonia are air-space consolidation, micronodular shadows, and groundglass opacities involving middle and lower lung fields (93). A study that included 112 patients with HM and recipients of HSCT having febrile neutropenia showed the following results: sensitivity of HR-CAT scans in detecting pneumonia in patients with febrile neutropenia and in recipients of HSCT was 87 and $88 \%$, specificity was 57 and $67 \%$, respectively, while negative predictive values were 88 and $97 \%$, respectively (92).

Fiber-optic bronchoscopy and bronchoalveolar lavage (BAL) have generally been considered as safe and accurate procedures for evaluation of patients having pulmonary infiltrates (94-96). The diagnostic yield of bronchoscopy in HSCT recipients may reach $75 \%$ and the results of BAL may change treatment in up to $51 \%$ of episodes of pneumonia (94-96).

Lung biopsies carry significant risk of morbidity and mortality in recipients of HSCT who are fragile, thrombocytopenic, and immunocompromised (94). Patients with HM and recipients of HSCT having pulmonary infiltrates or nodular lung lesions are at risk of treatment failure once conventional broad-spectrum antimicrobial therapies are used on empirical basis as filamentous fungi and MDR-GNB such as Pseudomonas aeruginosa and $S$. maltophilia may be involved etiologically (97). In these patients, pulmonary lesions should be characterized by HR-CAT scans, bronchoscopy, BAL, and even lung biopsies, if absolutely indicated (98). The choice of optimal biopsy should be individualized and guided by several patient and institutional factors. It is highly recommended to adopt interdisciplinary approach in order to optimize diagnosis and treatment (98).

In patients with HM and in recipients of HSCT, molecular diagnostic techniques such as PCR and nucleic acid amplification are promising tool for the rapid etiological diagnosis of pneumonia $(99,100)$. Their usefulness in the early diagnosis of viral and atypical pneumonia is well documented. However, their role in early detection of bacterial causes of pneumonia in patients with HM and in recipients of HSCT needs further evaluation and standardization of the diagnostic tools utilized $(99,100)$.

\section{TREATMENT OF S. MALTOPHILIA INFECTIONS}

Trimethoprim-sulfamethoxazole is still the drug of choice for S. maltophilia infections (Table 6) (4, 5, 14, 16, 67, 101, 102). Unfortunately, resistance to TMP-SMZ is increasingly reported and the rate of resistance is $8.3-9.1 \%(4,16,34,36,101,102)$. However, for successful eradication of S. maltophilia infections, TMP-SMZ must be given in a sufficient dose and at a proper frequency in order to produce adequate concentrations at the site(s) of infection. Determination of the appropriate dosing regimen requires optimum knowledge and utilization of the pharmacokinetics and pharmacodynamics of TMP-SMZ (103). Currently, the most effective antibiotics against $S$. maltophilia are: TMPSMZ, ticarcillin-clavulanic acid, minocycline, and the new fluoroquinolones such as moxifloxacin, ofloxacin, and levofloxacin. These agents can be used either alone or in various combinations $(36,64)$.

\section{Table 6 |This shows available and future therapeutic modalities for} S. maltophilia infections

\begin{tabular}{|c|c|}
\hline Therapeutic modality & Examples and details \\
\hline Monotherapies & $\begin{array}{l}\text {-TMP-SMZ: still the drug of choice } \\
\text { - Fluoroquinolones: ciprofloxacin, levofloxacin, } \\
\text { moxifloxacin, gatifloxacin, trovafloxacin, } \\
\text { ofloxacin. } \\
\text { - Ticarcillin-clavulanate } \\
\text { - Tetracyclines: minocycline, tigecycline } \\
\text { - Cephalosporines: ceftazidime. }\end{array}$ \\
\hline Combination therapies & $\begin{array}{l}\text {-TMP-SMZ and ticarcillin-clavulanate } \\
\text {-TMP-SMZ and ceftazidime } \\
\text {-TMP-SMZ and tigecycline } \\
\text { - Tigecycline and amikacin } \\
\text { - Colistin and tigecycline } \\
\text { - Aerosolized colistin and doxycycline } \\
\text { - MDR species: colistin and rifampicin or } \\
\text { colistin and TMP-SMZ }\end{array}$ \\
\hline $\begin{array}{l}\text { Targeted and future } \\
\text { therapies }\end{array}$ & $\begin{array}{l}\text { - Inhibitors of efflux pumps } \\
\text { - Inhibitors of } \beta \text {-lactamases } \\
\text { - Antimicrobial peptides } \\
\text { - Cationic compounds } \\
\text { - Bacteriophages } \\
\text { - Nanoemulsions } \\
\text { - Telavancin and colistin } \\
\text { - Plant oils and constituents of green tea }\end{array}$ \\
\hline
\end{tabular}

TMP-SMZ, trimethoprim-sulfamethoxazole; MDR, multidrug resistance.

Alternative therapies to TMP-SMZ include: (1) monotherapies including cephalosporins such as ceftazidime; fluoroquinolones such as ciprofloxacin, levofloxacin including the aerosolized form, moxifloxacin, gatifloxacin, and trovafloxacin; ticarcillinclavulanate and tetracyclines such as tigecycline or minocycline and (2) drug combinations such as: TMP-SMZ and ticarcillinclavulanate, TMP-SMZ and tigecycline, tigecycline and amikacin, colistin and tigecycline, telavancin and colistin, or aerosolized colistin and doxycycline (Table 6) (2, 4, 17, 101, 104, 105). Several recent studies have shown the efficacy of levofloxacin monotherapy as an alternative to treatment with TMP-SMZ $(37,53,106)$. Antimicrobials that are active against $S$. maltophilia should preferably be used in combinations, particularly once MDR S. maltophilia is isolated (42-48). In the treatment of infections caused by MDR $S$. maltophilia, colistin can be used in combination with rifampicin or TMP-SMZ (107).

The Fourth European Conference on Infections in Leukemia (ECIL-4, 2011) recommended the following targeted therapies for S. maltophilia infections in patients with leukemia and in recipients of HSCT: (1) TMP-SMZ, (2) fluoroquinolones such as ciprofloxacin and moxifloxacin, (3) ticarcillin-clavulanate, and (4) in seriously ill or neutropenic patients, combination therapy may be considered such as TMP-SMZ and ceftazidime or TMPSMZ and ticarcillin-clavulanate (41). Targeted therapy against MDR bacteria should be based on: (1) in vitro susceptibility data, (2) knowledge of the best treatment option against 
the particular species or phenotype of bacteria, (3) pharmacokinetic and pharmacodynamic data, and (4) careful assessment of risk-benefit balance (41). For infections due to GNB, antimicrobials should be preferably used in combination with other agents that remain active in vitro because of suboptimal efficacy in case of tigecycline and the risk of emergent resistance in case of fosfomycin. There is a growing problem of antimicrobial resistance among pathogens isolated from patients with HM and recipients of HSCT in many centers and this increasingly influences the choice of empirical antimicrobial therapy (41).

The standard therapy with TMP-SMZ may be difficult to administer in recipients of HSCT particularly those having cytopenias due to its myelosuppressive effects (14). In such patients, the following alternative antimicrobials can be used alone or in combinations: ceftazidime or ceftriaxone, ticarcillin-clavulanic acid, and levofloxacin or moxifloxacin $(5,14)$. An additional concern is that these patients receive plenty of other drugs such as cyclosporine-A, mycophenolate mofetil, triazoles, and antivirals that may predispose not only to more adverse effects but also to drug interactions (41). Therefore, use of specific antimicrobials should be cautiously considered. Antimicrobial stewardship aiming to minimize unnecessary broad-spectrum antibiotic use and its associated collateral damage and resistance selection is crucial in the current era of growing drug resistance (41). In patients with HM and in recipients of HSCT having infections caused by MDR S. maltophilia, IV colistin with dosage adjusted to renal function is relatively safe, even if given concomitantly with other nephrotoxic medications (108).

In patients with device-associated infections caused by S. maltophilia, fluoroquinolones particularly levofloxacin can be used to eradicate such infections once used in combination with other antimicrobials (109). In patients with VAP caused by MDR $S$. maltophilia, the following treatment options are safe and efficacious: (1) doxycycline and aerosolized colistin, and (2) IV colistin $(27,110,111)$. Despite the emergence of antimicrobial resistance, fluoroquinolone prophylaxis may be considered as an appealing option in recipients of HSCT. However, their usefulness in prophylaxis in allogeneic HSCT should be evaluated in randomized trials $(112,113)$.

\section{NEW AND FUTURE THERAPEUTIC MODALITIES}

New therapeutic strategies and future targeted therapies for $S$. maltophilia infections include the use of antimicrobial peptides, cationic compounds, bacteriophages, nanoemulsions, plant oils, constituents of green tea as well as inhibitors of $\beta$-lactamases and efflux pumps $(2,17)$.

The targeted therapies include bacterial efflux pumps inhibitors (BEPIs), inhibitors of $\beta$-lactamases, inhibitors of bacterial quorum sensing, antimicrobial peptides, bioengineered bacteriophage therapy, monoclonal antibodies directed against S. maltophilia, and antibodies to surface polysaccharides, which mediate biofilm formation and that are expressed during infection (Table 6) (114-124). Several families of BEPIs have been described (116). BEPIs have variable mechanisms of actions that include increasing intracellular concentrations of antibiotics that are expelled by efflux pumps, restoration of drug susceptibility of resistant clinical isolates, reduction of capability of acquired additional resistance, and reduction of intrinsic bacterial resistance to antimicrobials (114-116).

\section{PREVENTION OF S. MALTOPHILIA INFECTIONS}

Prevention of $S$. maltophilia infections can be achieved by: (1) health education of health-care personnel to prevent transmission and spread of this opportunistic pathogen, (2) observation of aqueous-associated environment and regular cleaning and disinfection of surfaces of medical instruments, (3) reinforcement of hand washing hygiene, (4) avoidance of using hospital tap water for bathing or cleaning of wounds, (5) discarding residual antibiotic solutions and contaminated fluids, (6) regular maintenance of hospital equipment and replacement of defective and worn parts, and (7) control of antimicrobial consumption (2, 17). Limitation of antimicrobial resistance and restriction of spread of the organism can be achieved by: appropriate antimicrobial selection, surveillance systems, and effective infection control measures (28).

\section{COURSE AND PROGNOSIS OF S. MALTOPHILIA INFECTIONS}

Morbidity and mortality associated with severe S. maltophilia infections are high due to the following reasons: (1) the organism is inherently resistant to antimicrobials, (2) antimicrobial resistance increases when the patient is colonized with the organism or given broad-spectrum antibiotics, and (3) the debilitated status of individuals acquiring the infection (6).

Risk factors for S. maltophilia infection-associated mortality include: (1) severely immunocompromised hosts, particularly those with underlying HM and recipients of HSCT, (2) septic shock, (3) organ failure, (4) bacteremia, (5) lung involvement such as pneumonia or pulmonary hemorrhage, (6) extensively drug-resistant organism, (7) exposure to carbapenems, (8) thrombocytopenia, and (9) high acute physiology and chronic health evaluation (APACHE) score in patients admitted to the ICU $(2,5$, 7-9, 18, 60, 125, 126).

In $S$. maltophilia-associated infections, successful outcome is associated with administration of antibiotics to which the organism is susceptible, removal of infected devices such as CVCs, recovery of bone marrow function, taking enough preventive and isolation measures, and having a high index of suspicion $(2,8)$.

\section{CONCLUSION}

Stenotrophomonas maltophilia is a globally emerging pathogen that causes serious infectious complications in immunocompromised patients in particular. It also exhibits a wide range of drug resistance mechanisms and this complicates the management of infections caused by this GNB. It is essential to utilize the available antimicrobials appropriately, use novel agents to which the organism is susceptible, and to strictly apply infection control measures in order to decrease the incidence of infections caused by $S$. maltophilia.

\section{REFERENCES}

1. Denton M, Kerr KG. Microbiological and clinical aspects of infection associated with Stenotrophomonas maltophilia. Clin Microbiol Rev (1998) 11(1):57-80.

2. Brooke JS. Stenotrophomonas maltophilia: an emerging global opportunistic pathogen. Clin Microbiol Rev (2012) 25(1):2-41. doi:10.1128/CMR.00019-11 
3. Lewis SS, Zaas A. Stenotrophomonas maltophilia. In: Sexton DJ, Bloom A, editors. UpToDate (2014). Topic 13961, version 8.0: pages: 1-8. Topic last updated: Jan 28, 2014.

4. Falagas ME, Valkimadi P-E, Huang Y-T, Matthaiou DK, Hsueh P-R. Therapeutic options for Stenotrophomonas infections beyond co-trimoxazole: a systematic review. J Antimicrob Chemother (2008) 62:889-94. doi:10.1093/jac/dkn301

5. Al-Anazi KA, Al-Jasser AM. Bacteremia due to Stenotrophomonas maltophilia in patients with hematological malignancies. Kuwait Med J (2006) 38(3):214-9.

6. Micozzi A, Venditti M, Monaco M, Friedrich A, Taglietti F, Santilli S, et al. Bacteremia due to Stenotrophomonas maltophilia in patients with hematologic malignancies. Clin Infect Dis (2000) 31:705-11. doi:10.1086/314043

7. Tada K, Kurosawa S, Hiramoto N, Okinaka K, Ueno N, Asakura Y, et al. Stenotrophomonas maltophilia infection in hemotopoietic SCT recipients: high mortality due to pulmonary hemorrhage. Bone Marrow Transplant (2013) 48:74-9. doi:10.1038/bmt.2012.87

8. Araoka H, Fujii T, Izutsu K, Kimura M, Nishida A, Ishiwata K, et al. Rapidly progressive pulmonary hemorrhage caused by Stenotrophomonas maltophilia in hematologic malignancy. Transpl Infect Dis (2012) 14(4):355-63. doi:10. 1111/j.1399-3062.2011.00710.x

9. Labarca JA, Leber AL, Kern VL, Territo MC, Brankovic LE, Bruckner DA, et al. Outbreak of Stenotrophomonas maltophilia bacteremia in allogeneic bone marrow transplant patients. Role of severe neutropenia and mucositis. Clin Infect Dis (2000) 30:195-7. doi:10.1086/313591

10. Klausner JD, Zukerman C, Limaye AP, Corey L. Outbreak of Stenotrophomonas maltophilia bacteremia among patients undergoing bone marrow transplantation: association with faulty replacement of hand washing soap. Infect Control Hosp Epidemiol (1999) 20(11):756-8. doi:10.1086/501578

11. Safdar A, Rolston KV. Stenotrophomonas maltophilia: changing spectrum of a serious bacterial pathogen in patients with cancer. Clin Infect Dis (2007) 45:1602-9. doi:10.1086/522998

12. Apisarnthanarak A, Mayfield JL, Garison T, McLendon PM, DiPerso JF, Fraser VJ, et al. Risk factors for Stenotrophomonas maltophilia bacteremia in oncology patients: a case-control study. Infect Control Hosp Epidemiol (2003) 24(4):269-74. doi:10.1086/502197

13. Yeshurun M, Grafter-Gvili A, Thaler M, Keller N, Nagler A, Shimoni A. Clinical characteristics of Stenotrophomonas maltophilia infection in hematopoietic stem cell transplantation recipients: a single center experience. Infection (2010) 38(3):211-5. doi:10.1007/s15010-010-0023-2

14. Chaplow R, Palmer B, Heyderman R, Moppett J, Marks DI. Stenotrophomonas maltophilia bacteremia in 40 hematology patients: risk factors, therapy and outcome. Bone Marrow Transplant (2010) 45:1109-10. doi:10.1038/bmt.2009.274

15. Caylan R, Yilmaz G, Sucu N, Bayraktar O, Aydin K, Kaklikkaya N, et al. Nosocomial Stenotrophomonas maltophilia infections in a university hospital. Mikrobiyol Bul (2005) 39(1):25-33.

16. Muder RR. Optimizing therapy for Stenotrophomonas maltophilia. Semin Respir Crit Care Med (2007) 28(6):672-7. doi:10.1055/s-2007-996414

17. Looney WJ, Narita M, Mühleman K. Stenotrophomonas maltophilia: an emerging opportunist human pathogen. Lancet Infect Dis (2009) 9:213-23. doi:10. 1016/S1473-3099(09)70083-0

18. Lai CH, Chi CY, Chen HP, Chen TL, Lai CJ, Fung CP, et al. Clinical characteristics and prognostic factors of patients with Stenotrophomonas maltophilia bacteremia. J Microbiol Immunol Infect (2004) 37(6):350-8.

19. Boktour M, Hanna H, Ansari S, Bahna B, Hachem R, Tarrand J, et al. Central venous catheter and Stenotrophomonas maltiphilia bacteremia in cancer patients. Cancer (2006) 106:1967-73. doi:10.1002/cncr.21846

20. Fletcher S. Catheter-related bloodstream infection. Contin Educ Anaesth Crit Care Pain (2005) 5(2):49-51. doi:10.1093/bjaceaccp/mki011

21. Mermel LA, Allon M, Bouza E, Craven DE, Flynn P, O'Grady NP. Clinical practice guidelines for the diagnosis and management of intravascular catheterrelated infections: 2009 update by the Infections Disease Society of America. Clin Infect Dis (2009) 49:1-45. doi:10.1086/599376

22. Hanna H, Afif C, Alakech B, Boktour M, Tarrand J, Hachem R, et al. Central venous catheter-related bacteremia due to Gram-negative bacilli: significance of catheter removal in preventing disease. Infect Control Hosp Epidemiol (2004) 25(8):646-9. doi:10.1086/502455

23. Dias MBS, Habert AB, Burrasca V, Stempliuk V, Ciolli A, Araujo RE, et al. Salvage of long-term central venous catheters during an outbreak of Pseudomonas putida and Stenotrophomonas maltophilia infections associated with contaminated heparin catheter-lock solution. Infect Control Hosp Epidemiol (2008) 29(2):125-30. doi:10.1086/526440

24. Crnich CJ, Maki DG. The promise of novel technology for the prevention of intravascular device-related bloodstream infection. II. Long-term devices. Clin Infect Dis (2002) 34:1362-8. doi:10.1086/340105

25. Shiratori S, Wakasa K, Okada K, Sugita J, Akizawa K, Shigematsu A, et al. Stenotrophomonas maltophilia infection during allogeneic hematopoietic stem cell transplantation: a single-center experience. Clin Transplant (2014) 28(6):656-61. doi:10.1111/ctr.12356

26. Nseir S, Di Pompeo C, Brisson H, Dewavrin F, Tissier S, Diarra M, et al. Intensive care unit-acquired Stenotrophomonas maltophilia: incidence, risk factors and outcome. Crit Care (2006) 10:R143. doi:10.1186/cc4943

27. Wood GC. Treatment of recurrent Stenotrophomonas ventilator-associated pneumonia with doxycycline and aerosolized colistin. Ann Pharmacother (2010) 44(10):1665-8. doi:10.1345/aph.1P217

28. Mendoza DL, Darin M, Waterer GW, Wunderink RG. Update on Stenotrophomonas maltophilia infection in the ICU. Clin Pulm Med (2007) 14(1):17-22. doi:10.1097/01.cpm.0000252835.11240.42

29. Mayer SA, Pastores S, Adamu M, Halpern NA, Young JW, Jakubowski A. Outcomes of adult allogeneic hematopoietic stem cell transplant patients admitted to the intensive care unit in the peri-transplant period. Chest (2008) 134:60003. doi:10.1378/chest.134.4_MeetingAbstracts.p60003

30. Mori M, Kitagawa T, Sasaki Y, Yamamoto K, Onaka T, Yonezawa A, et al. Lethal pulmonary hemorrhage caused by Stenotrophomonas maltophilia pneumonia in a patient with acute myeloid leukemia. Kansenshogaku Zasshi (2012) 86(3):300-5. doi:10.11150/kansenshogakuzasshi.86.300

31. Gupta S, Jain A, Werneke CL, Gupta A, Shannon VR, Morice RC, et al. Outcome of alveolar hemorrhage in hematopoietic stem cell transplant recipients. Bone Marrow Transplant (2007) 40:71-8. doi:10.1038/sj.bmt.1705695

32. Mori M, Tsunemine H, Imada K, Ito K, Kodaka T, Takahashi T. Lifethreatening hemorrhagic pneumonia caused by Stenotrophomonas maltophilia in the treatment of hematologic diseases. Ann Hematol (2014) 93(6):901-11. doi:10.1007/s00277-014-2028-x

33. Wang W-S, Liu C-P, Lee C-M, Huang F-Y. Stenotrophomonas maltophilia bacteremia in adults: four years' experience in a medical center in northern Taiwan. J Microbiol Immunol Infect (2004) 37:359-65.

34. Naidu P, Smith S. A review of 11 years of Stenotrophomonas maltophilia blood isolates at a tertiary care institute in Canada. Can J Infect Dis Med Microbiol (2012) 23(4):165-9.

35. Senol E, Des Jardin J, Stark PC, Barefoot L, Syndman DR. Attributable mortality of Stenotrophomonas maltophilia bacteremia. Clin Infect Dis (2002) 34: 1653-6. doi:10.1086/340707

36. Wu P-S, Lu C-Y, Chang L-Y, Hsueh P-R, Lee P-S, Chen J-M, et al. Stenotrophomonas maltophilia bacteremia in pediatric patients - a 10 -year analysis. J Microbiol Immunol Infect (2006) 39:144-9.

37. Hotta G, Matsumura Y, Kato K, Nakano S, YunoKi T, Yamamoto M, et al. Risk factors and clinical characteristics of Stenotrophomonas maltophilia bacteremia: a comparison with bacteremia due to other glucose-non fermenters. Kansenshogaku Zasshi (2013) 87(5):596-602.

38. Gales AC, Jones RN, Forward KR, Linares J, Sader HS, Verhoef J. Emerging importance of multidrug-resistant Acinetobacter species and Stenotrophomonas maltophilia as pathogens in seriously ill patients: geographic patterns, epidemiological features and trends in the SENTRY Antimicrobial Surveillance Program (1997-1999). Clin Infect Dis (2001) 32(Suppl 2):104-13. doi:10.1086/ 320183

39. Memish ZA, Shibl AF, Kambal AM, Ohaly YA, Ishaq A, Livermore DM. Antimicrobial resistance among non-fermenting Gram-negative bacteria in Saudi Arabia. J Antimicrob Chemother (2012) 67:1701-5. doi:10.1093/jac/dks091

40. Nicodemo AC, Paez JI. Antimicrobial therapy for Stenotrophomonas maltophilia infections. Eur J Clin Microbiol Infect Dis (2007) 26(4):229-37. doi:10.1007/s10096-007-0279-3

41. Averbuch D, Cordonnier C, Livermore DM, Mikulska M, Orasch C, Viscoli C, et al. on behalf of ECIL 4, a joint venture of EBMT, EORTC, ICHS, ESGICH/ESCMID and ELN. Targeted therapy against multi-resistant bacteria in leukemia and hematopoietic stem cell transplant recipients: guidelines of the 4th Europian Conference on Infections in Leukemia (ECIL4, 2011). Haematologica (2013) 98(12):1836-47. doi:10.3324/haematol.2013. 091330 
42. Zhanel GG, Adam HJ, Baxter MR, Fuller J, Nichol KA, Denisuik AJ, et al. Antimicrobial susceptibility of 22,746 pathogens from Canadian hospitals: results of the CANWARD 2007-11 Study. J Antimicrob Chemother (2013) 68(Suppl 1):i7-22. doi:10.1093/jac/dkt022

43. Brooke SJ. New strategies against Stenotrophomonas maltophilia: a serious worldwide intrinsically drug-resistant opportunistic pathogen. Expert Rev Anti Infect Ther (2014) 12(1):1-4. doi:10.1586/14787210.2014.864553

44. Gulmez D, Cakar A, Sener B, Karakaya J, Hascelik G. Comparison of different antimicrobial susceptibility testing methods for Stenotrophomonas maltophilia and results of synergy testing. J Infect Chemother (2010) 16(5):322-8. doi:10.1007/s10156-010-0068-2

45. Liaw S-J, Teng L-J, Hsueh P-R, Ho S-W, Luh T-T. In vitro activities of antimicrobial combinations against clinical isolates of Stenotrophomonas maltophilia. J Formos Med Assoc (2002) 101(7):495-501.

46. Abbott IJ, Slavin MA, Turnidge JD, Thursky KA, Worth LJ. Stenotrophomonas maltophilia: emerging disease patterns and challenges for treatment. Expert Rev Anti Infect Ther (2011) 9(4):471-88. doi:10.1586/eri.11.24

47. Samonis G, Karageogopoulos DE, Maraki S, Levis P, Dimopoulou D, Spernovasillis NA, et al. Stenotrophomonas maltophilia infections in a general hospital: patient characteristics, antimicrobial susceptibility and treatment outcome PLoS One (2012) 7(5):e37375. doi:10.1371/journal.pone.0037375

48. Betriu C, Sänchez A, Palau ML, Gomez M, Picazo JJ. Antibiotic resistance surveillance of Stenotrophomonas maltophilia, 1993-1999. J Antimicrob Chemother (2001) 48:152-4. doi:10.1093/jac/48.1.152

49. Alonso A, Martinez JL. Multiple antibiotic resistance in Stenotrophomonas maltophilia. Antimicrob Agents Chemother (1997) 41(5):1140-2.

50. Li X-Z, Zhang L, Poole K. SmeC, an outer membrane multidrug efflux protein of Stenotrophomonas maltophilia. Antimicrob Agents Chemother (2002) 46(2):333-43. doi:10.1128/AAC.46.2.333-343.2002

51. Li X-Z, Zhang L, Mckay GA, Poole K. Role of the acetyltransferase AAC(6')Iz modifying enzyme in aminoglycoside resistance in Stenotrophomonas maltophilia. J Antimicrob Chemother (2003) 51:803-11. doi:10.1093/jac/dkg148

52. Fihman V, Le Monnier A, Corvec S, Jaureguy F, Tankovic J, Jacquir H, et al. Stenotrophomonas maltophilia - the most worrisome threat among unusual non-fermentative Gram-negative bacilli from hospitalized patients: a prospective multicenter study. J Infect (2012) 64(4):391-8. doi:10.1016/j.jinf. 2012.01.001

53. Wang YL, Scipione MR, Dubrovskaya Y, Papadopoulos J. Monotherapy with fluoroquinolones or trimethoprim-sulfamethoxazole for treatment of Stenotrophomonas maltophilia infections. Antimicrob Agents Chemother (2014) 58(1):176-82. doi:10.1128/AAC.01324- 13

54. Chung H-S, Hong SG, Kim YR, Shin KS, Whang DH, Ahn JY, et al. Antimicrobial susceptibility of Stenotrophomonas maltophilia isolates from Korea, and the activity of antimicrobial combinations against the isolates. J Korean Med Sci (2013) 28:62-6. doi:10.3346/jkms.2013.28.1.62

55. Rhee J-Y, Choi JY, Choi M-J, Song J-H, Peck KR, Ko KS. Distinct groups and antimicrobial resistance of clinical Stenotrophomonas complex isolates from Korea. J Med Microbiol (2013) 62:748-53. doi:10.1099/jmm.0.053355-0

56. Naeem T, Absar M, Somily AM. Antibiotic resistance among clinical isolates of Stenotrophomonas maltophilia at a teaching hospital in Riyadh, Saudi Arabia. J Ayub Med Coll Abbottabad (2012) 24(2):30-3.

57. Toleman MA, Bennett PM, Bennett DMC, Jones RN, Walsh TR. Global emergence of trimethoprim/sulfamethoxazole resistance in Stenotrophomonas maltophilia mediated by acquisition of sul genes. Emerg Infect Dis (2007) 13(4):559-65. doi:10.3201/eid1304.061378

58. Song JH, Sung JY, Kwon KC, Park JW, Cho HH, Shin SY, et al. Analysis of acquired resistance genes in Stenotrophomonas maltophilia. Korean J Lab Med (2010) 30(3):295-300. doi:10.3343/kjlm.2010.30.3.295

59. Alonso A, Sanchez P, Martinez JL. Stenotrophomonas maltophilia D457R contains a cluster of genes from Gram-positive bacteria involved in antibiotic and heavy metal resistance. Antimicrob Agents Chemother (2000) 44(7):1778-82. doi:10.1128/AAC.44.7.1778-1782.2000

60. Tan CK, Liaw SJ, Yu CJ, Teng LJ, Hsueh PR. Extensively drug-resistant Stenotrophomonas maltophilia in a tertiary care hospital in Taiwan: microbiologic characteristics, clinical features and outcome. Diagn Microbiol Infect Dis (2008) 60(2):205-10.

61. McKay GA, Woods DE, MacDonald KL, Poole K. Role of phosphoglucomutase of Stenotrophomonas maltophilia in lipopolysaccharide biosynthesis, virulence and antibiotic resistance. Infect Immunol (2003) 71(6):3068-75. doi:10.1128/IAI.71.6.3068-3075.2003

62. Zhang L, Li X-Z, Poole K. SmeDEF multidrug efflux pump contributes to intrinsic multidrug resistance in Stenotrophomonas maltophilia. Antimicrob Agents Chemother (2001) 45(12):3497-503. doi:10.1128/AAC.45.12.34973503.2001

63. Metan G, Uzun O. Impact of initial antimicrobial therapy in patients with bloodstream infections caused by Stenotrophomonas maltophilia. Antimicrob Agents Chemother (2005) 49(9):3980-1. doi:10.1128/AAC.49.9.3980-3981. 2005

64. Gordon NC, Wareham DW. Novel variants of the Smqnr family of quinolone resistance genes in clinical isolates of Stenotrophomonas maltophilia. J Antimicrob Chemother (2010) 65:483-9. doi:10.1093/jac/dkp476

65. Karunanidhi A, Thomas R, van Belkum A, Neela V. In vitro antimicrobial and anitibiofilm activities of chlorogenic acid against clinical isolates of Stenotrophomonas maltophilia including the trimethoprim/sulfamethoxazole resistant strain. Biomed Res Int (2013) 2013:392058. doi: $10.1155 / 2013 / 392058$

66. Di Bonaventura G, Spedicato I, D'Antoniop D, Robuffo I, Piccolomini R. Biofilm formation by Stenotrophomonas maltophilia: modulation by quinolones, trimethoprim-sulfamethoxizole and ceftazidime. Antimicrob Agents Chemother (2004) 48(1):151-60. doi:10.1128/AAC.48.1.151-160.2004

67. Crossman LC, Gould VC, Dow JM, Vernikos GS, Okazaki A, Sebaihia $\mathrm{M}$, et al. The complete genome, comparative and functional analysis of Stenotrophomonas maltophilia reveals an organism heavily shielded by drug resistance determinants. Genome Biol (2008) 9(4):1-13. doi:10.1186/gb-20089-4-r74

68. Zhang L, Morrison M, Cuiv PO, Evans P, Rickard CM. Genome sequence of Stenotrophomonas maltophilia strain AU 12-09 isolated from an intravascular catheter. Genome Announc (2013) 1(3):e00195-00203. doi:10.1128/genomeA. 00195- 13

69. Chen C-H, Huang C-C, Chung T-C, Hu R-M, Huang Y-W, Yang T-C. Contribution of resistance-nodulation-division efflux pump operon $S m e \mathrm{U}_{1}-\mathrm{V}-\mathrm{W}-\mathrm{U}_{2}$ $\mathrm{X}$ to multidrug resistance of Stenotrophomonas maltophilia. Antimicrob Agents Chemother (2011) 55(2):5826-33. doi:10.1128/AAC.00317-11

70. Chang Y-C, Tsai M-J, Huang Y-W, Chung T-C, Yang T-C. SmQnrR, a DeoRtype transcriptional regulator, negatively regulates the expression of Smqnr and SmtcrA in Stenotrophomonas maltophilia. J Antimicrob Chemother (2011) 66:1024-8. doi:10.1093/jac/dkr049

71. Lin C-W, Huang Y-W, Hu R-M, Yang T-C. Sme OP-tolCsm efflux pump contributes to the multidrug resistance of Stenotrophomonas maltophilia. Antimicrob Agents Chemother (2014) 58(4):2405-8. doi:10.1128/AAC.01974- 13

72. Huang Y-W, Hu M-R, Yang T-C. Role of the pcm-tolCsm operon in the multidrug resistance of Stenotrophomonas maltophilia. J Antimicrob Chemother (2013) 68(9):1987-93. doi:10.1093/jac/dkt148

73. Hu LF, Chang X, Ye Y, Wang ZX, Shao YB, Shi W, et al. Stenotrophomonas maltophilia resistance to trimethoprim/sulfamethoxazole mediated by acquisition of sul and dfrA genes in a plasmid-mediated class/integron. Int J Antimicrob Agents (2011) 37(3):230-4. doi:10.1016/j.ijantimicag.2010.10.025

74. Toleman MA, Bennett PM, Walsh TR. ISCR elements: novel gene-capturing systems of the 21st century? Microbiol Mol Biol Rev (2006) 70(3):296-316. doi:10.1128/MMBR.00048-05

75. Neela V, Rankouhi SZ, van Belkum A, Goering RV, Awang R. Stenotrophomonas maltophilia in Malaysia: molecular epidemiology and trimethoprimsulfamethoxazole resistance. Int J Infect Dis (2012) 16(8):e603-7. doi:10.1016/ j.ijid.2012.04.004

76. Turrientes MC, Baquero MR, Sanchez MB, Valdezate S, Escudero E, Berg $\mathrm{G}$, et al. Polymorphic mutation frequencies of clinical and environmental Stenotrophomonas maltophilia populations. Appl Environ Microbiol (2010) 76(6):1746-58. doi:10.1128/AEM.02817-09

77. Mukherjee P, Roy P. Cloning, sequencing and expression of novel trichloroethylene degradation genes from Stenotrophomonas maltophilia PM102: a case of gene duplication. J Bioremed Biodegradation (2013) 4(2). doi:10.4172/21556199.1000177

78. Mukherjee P, Roy P. Copper enhanced monooxygenase activity and FT-IR spectroscopic characterization of biotransformation products in trichloroethylene degrading bacterium: Stenotrophomonas maltophilia PM102. Biomed Res Int (2013) 2013:723680. doi:10.1155/2013/723680 
79. Mukherjee P, Roy P. Purification and identification of trichloroethylene induced proteins from Stenotrophomonas maltophilia PM102 by immunoaffinity-chromatography and MALDI-TOF mass spectrometry. Springer Plus (2013) 2:207. doi:10.1186/2193-1801-2-207

80. Hernandez A, Ruiz FM, Romero A, Martinez JL. The binding of triclosan to Sme T, the repressor of the multidrug efflux pump Sme DEF, induces antibiotic resistance in Stenotrophomonas maltophilia. PLoS Pathog (2011) 7(6):e1002103. doi:10.1371/journal.ppat.1002103

81. Kempf VA, Mandle T, Schumacher U, Schäfer A, Autenrieth IB. Rapid detection and identification of pathogens in blood cultures by fluorescence in site hybridization and flow cytometry. Int J Med Microbiol (2005) 295(1):47-55. doi:10.1016/j.ijmm.2004.12.006

82. Gallo SW, Ramos PL, Sanchez Ferreira CA, de Oliveira SD. A specific polymerase chain reaction method to identify Stenotrophomonas maltophilia. Mem Inst Oswaldo Cruz (2013) 108(3):390-1. doi:10.1590/S007402762013000300020

83. Nakamura A, Sugimoto Y, Ohishi K, Sugawara Y, Fujieda A, Monma F, et al. Diagnostic value of PCR analysis of bacteria and fungi from blood in empirictherapy-resistant febrile neutropenia. J Clin Microbiol (2010) 48(6):2030-6. doi:10.1128/JCM.01700-09

84. Menezes LC, Rocchetti TT, de Castro Bauab K, Cappellano P, Quiles MG, Carlesse F, et al. Diagnosis by real-time polymerase chain reaction of pathogens and antimicrobial resistance genes in bone marrow transplant patients with bloodstream infections. BMC Infect Dis (2013) 13:166. doi:10.1186/1471-2334-13166

85. Xu J, Moore JE, Miller BC, Alexander HD, McClurg R, Morris TC, et al. Improved laboratory diagnosis of bacterial and fungal infections in patients with hematological malignancies using PCR and ribosomal RNA sequence analysis. Leuk Lymphoma (2004) 45(8):1637-41. doi:10.1080/ 10428190410001667695

86. Lamoth F, Jaton K, Prod'hom G, Senn L, Bille J, Calandra T, et al. Multiplex blood PCR in combination with blood cultures for improvement of microbiological documentation of infection in febrile neutropenia. J Clin Microbiol (2010) 48(10):3510-6. doi:10.1128/JCM.00147-10

87. von Lilienfeld-Toal M, Lehmann LE, Raadts AD, Hahn-Ast C, Orloop KS, Marklein G, et al. Utility of a commercially available multiplex real-time PCR assay to detect bacterial and fungal pathogens in febrile neutropenia. J Clin Microbiol (2009) 47(8):2405-10. doi:10.1128/JCM.00491-09

88. Bravo D, Blanquer J, Tormo M, Aguillar G, Borras R, Solano C, et al. Diagnostic accuracy and potential clinical value of the LightCycler SeptiFast assay in the management of bloodstream infections occurring in neutropenic and critically ill patients. Int J Infect Dis (2011) 15(5):e326-e331. doi:10.1016/j.ijid.2011.01.003

89. Kassel TWK, Ryan LKL, Li A. Computed tomography features of Stenotrophomonas maltophilia pneumonia in patients with neutropenic fever: report of two cases. Multidiscip Respir Med (2013) 8:14. doi:10.1186/20496958-8-14

90. Makis W, Ciarallo A, Rush C, Hickeson M. Infectious and inflammatory complications of surgical management of cancer patients imaged with $18 \mathrm{~F}$ FDG PET/CT: a pictoral assay. Clin Imaging (2013) 37:669-79. doi:10.1016/j. clinimag.2013.02.003

91. Heussel CP, Kauczor H-U, Hessel G, Fischer B, Mildenberger P, Thelen M. Early detection of pneumonia in febrile neutropenic patients: use of thin-section CT. Am J Radiol (1997) 169:1347-53.

92. Heussel CP, Kauczor H-U, Heussel GE, Fisher B, Begrich M, Mildenberger P, et al. Pneumonia in febrile neutropenic patients and in bone marrow and blood stem cell transplant recipients: use of high resolution computed tomography. J Clin Oncol (1999) 17(3):796-805.

93. Coelho LO, Gasparetto TD, Escuissato DL, Marchiori E. Bacterial pneumonia following bone marrow transplantation: HRCT findings. J Bras Pneumol (2009) 35(5):431-5. doi:10.1590/S1806-37132009000500007

94. Forslow U, Remberger M, Nordlander A, Mattsson J. The clinical importance of bronchoalveolar lavage in allogeneic SCT patients with pneumonia. Bone Marrow Transplant (2010) 45:945-50. doi:10.1038/bmt.2009.268

95. Hofmeister CC, Czerlanis C, Forsythe S, Stiff PJ. Retrospective utility of bronchoscopy after hematopoietic stem cell transplant. Bone Marrow Transplant (2006) 38:693-8. doi:10.1038/sj.bmt.1705505
96. Shannon VR, Andersson BS, Lei X, Champlin RE, Kontoyiannis DP. Utility of early versus late fiberoptic bronchoscopy in the evaluation of new pulmonary infiltrates following hematopoietic stem cell transplantation. Bone Marrow Transplant (2010) 45:647-55. doi:10.1038/bmt.2009.203

97. Maschmeyer G, Beinert T, Buchheidt D, Cornely OA, Einsele H, Heinz W, et al. Diagnosis and antimicrobial therapy of lung infiltrates in febrile neutropenic patients: guidelines for the infectious diseases working party of the German Society of Hematology and Oncology. Eur J Cancer (2009) 45(14):2462-72. doi:10.1016/j.ejca.2009.05.001

98. Wingard JR, Hiemenz JW, Jantz MA. How I manage pulmonary nodular lesions and nodular infiltrates in patients with hematologic malignancies or undergoing hematopoietic cell transplantation. Blood (2012) 120(9):1791-800. doi:10.1182/blood-2012-02-378976

99. van Elden LJ, van Kraaij MG, Nijhuis M, Hendriksen KAW, Dekker AW, Rozenberg-Arska M. Polymerase chain reaction is more sensitive than viral culture and antigen testing for the detection of respiratory viruses in adults with hematological cancer and pneumonia. Clin Infect Dis (2002) 34:177-83. doi: $10.1086 / 338238$

100. Murdoch DR. Nucleic acid amplification tests for the diagnosis of pneuomonia. Clin Infect Dis (2003) 36:1162-70. doi:10.1086/374559

101. Penzak SR, Abate BJ. Stenotrophomonas (xanthomonas) maltophilia: a multidrug-resistant nosocomial pathogen. Pharmacotherapy (1997) 17(2):293-301.

102. Al-Jasser AM. Stenotrophomonas maltophilia resistant to trimethoprimsulfamethoxazole: an increasing problem. Ann Clin Microbiol Antimicrob (2006) 5:23. doi:10.1186/1476-0711-5-23

103. Brown GR. Cotrimoxazole-optimal dosing in the critically ill. Ann Intens Care (2014) 4:13. doi:10.1186/2110-5820-4-13

104. Church D, Lloyed T, Peirano G, Pitout J. Antimicrobial susceptibility and combination testing of invasive Stenotrophomonas maltophilia isolates. Scand J Infect Dis (2013) 45(4):265-70. doi:10.3109/00365548.2012.732240

105. Hornsey M, Longshaw C, Phee L, Wareham DW. In vitro activity of telavancin in combination with colistin versus Gram-negative bacterial pathogens. Antimicrob Agents Chemother (2012) 56(6):3080-5. doi:10.1128/ AAC.05870-11

106. Cho SY, Kang CI, Kim J, Ha YE, Chung DR, Lee NY, et al. Can levofloxacin be a useful alternative to trimethoprim- sulfamethoxazole for treating Stenotrophomonas maltophilia bacteremia? Antimicrob Agents Chemother (2014) 58(1):581-3. doi:10.1128/AAC.01682-13

107. Giamarellos-Bourboulis EJ, Kamesis L, Giamarellou H. Synergy of colistin with rifampicin and trimethoprim-sulfamethoxazole on multidrug-resistant Stenotrophomonas maltophilia. Diagn Microbiol Infect Dis (2002) 44(3): 259-63. doi:10.1016/S0732-8893(02)00443-1

108. Avenbuch D, Horwitz E, Strahilevitz J, Stepenski P, Goldschmidt N, Gatt ME, et al. Colistin is relatively safe in hematological malignancies and hematopoietic stem cell transplantation patients. Infection (2013) 41(5):991-7. doi:10. 1007/s15010-013-0471-6

109. Passerini de Rossa B, Garcia C, Calenda M, Vay C, Franco M. Activity of levofloxacin and ciprofloxacin on biofilms and planktonic cells of Stenotrophomonas maltophilia isolates from patients with device-associated infections. Int J Antimicrob Agents (2009) 34(3):260-4. doi:10.1016/j. ijantimicag.2009.02.022

110. Ioannides K, Myrianthefs P, Baltopoulos G. Colistin as a first choice antibiotic for the initial empiric antimicrobial therapy of ventilator-associated pneumonia. Eur Respir J (2007) 30(6):1234-5. doi:10.1183/09031936.00102207

111. Florescu DF, Qiu F, McCartan MA, Mindru C, Fey PD, Kalil AC. What is the efficacy and safety of colistin for the treatment of ventilator-associated pneumonia? A systematic review and meta-analysis. Clin Infect Dis (2012) 54(5):670-80. doi:10.1093/cid/cir934

112. Busca A, Cavecchia I, Locatelli F, D'Ardia S, De Rosa FG, Marmont F, et al. Bloodstream infections after allogeneic stem cell transplantation: a singlecenter experience with the use of levofloxacin prophylaxis. Transpl Infect Dis (2012) 14(1):40-8. doi:10.1111/j.1399-3062.2011.00650.x

113. Bonadio M, Morelli G, Mori S, Riccioni R, Papineschi F, Petrini F. Fluoroquinolone resistance in hematopoietic stem cell transplant recipients with infectious complications. Biomed Pharmacother (2005) 59(9):511-6. doi:10. 1016/j.biopha.2005.06.008 
114. Mahmoud A, Chevalier J, Alibert-Franco S, Kern WV, Pages J-M. Antibiotic efflux pumps in Gram-negative bacteria: the inhibitor response strategy. J Antimicrob Chemother (2007) 59:1223-9. doi:10.1093/jac/ dk1493

115. Zechini B, Versace I. Inhibitors of multidrug resistant efflux systems in bacteria. Recent Pat Antiinfect Drug Discov (2009) 4(1):37-50. doi:10.2174/ 157489109787236256

116. Pages JM, Sandrine AF, Mahmoud A, Bolla JM, Davin-Regli A, Chevalier J, et al. Efflux pumps of Gram-negative bacteria, a new target for new molecules. Curr Top Med Chem (2010) 10(18):1848-57.

117. Leitner I, Nemeth J, Feurstein T, Abrahim A, Matzneller P, Lagler H, et al. The third-generation P-glycoprotein inhibitor tariquidar may overcome multidrug resistance by increasing intracellular drug concentration. J Antimicrob Chemother (2011) 66:834-9. doi:10.1093/jac/dkq526

118. Skurnik D, Davis MR Jr., Benedetti D, Moravec KL, Cywes-Bently C, Roux $\mathrm{D}$, et al. Targeting pan-resistant bacteria with antibodies to a broadly conserved surface polysaccharide expressed during infection. J Infect Dis (2012) 205:1709-18. doi:10.1093/infdis/jis254

119. Devocelle M. Targeted antimicrobial peptides. Front Immun (2012) 3:309. doi:10.3389/fimmu.2012.00309

120. Viertel TM, Ritter K, Horz H-P. Viruses versus bacteria-novel approaches to phage therapy as a tool against multidrug-resistant pathogens. J Antimicrob Chemother (2014) 69(9):2326-36. doi:10.1093/jac/dku173

121. AboZahra R. Quorum sensing and interspecies interactions in Stenotrophomonas maltophilia. Br Microbiol Res J (2013) 3(3):414-22. doi:10.1099/mic.0.026641-0

122. Bhardwaj AK, Vinothkumar K, Rajpara N. Bacterial quorum sensing inhibitors: attractive alternatives for control of infectious pathogens showing multiple drug resistance. Recent Pat Antiinfect Drug Discov (2013) 8(1):68-83. doi:10.2174/1574891X11308010012
123. Ronald PC. Small protein-mediated quorum sensing in a Gram-negative bacterium: novel targets for control of infectious disease. Discov Med (2011) 12(67):461-70.

124. Smalls FE. Development of an anti-Stenotrophomonas maltophilia Immunoglobulin-G (IgG) that Prevents Iron Transport in Gram-Negative Bacteria. Atlanta University Center (2009). 76 p. Available from: http:/digitalcommons.auctr.edu/dissertation/76

125. Garazi M, Singer C, Tai J, Ginocchio CC. Bloodstream infections caused by Stenotrophomonas maltophilia: a seven-year review. J Hosp Infect (2012) 81(2):114-8. doi:10.1016/j.jhin.2012.02.008

126. Paez JI, Costa SF. Risk factors associated with mortality of infections caused by Stenotrophomonas maltophilia: a systematic review. J Hosp Infect (2008) 70(2):101-8. doi:10.1016/j.jhin.2008.05.020

Conflict of Interest Statement: The authors declare that the research was conducted in the absence of any commercial or financial relationships that could be construed as a potential conflict of interest.

Received: 10 April 2014; accepted: 11 August 2014; published online: 25 August 2014. Citation: Al-Anazi KA and Al-Jasser AM (2014) Infections caused by Stenotrophomonas maltophilia in recipients of hematopoietic stem cell transplantation. Front. Oncol. 4:232. doi: 10.3389/fonc.2014.00232

This article was submitted to Hematology Oncology, a section of the journal Frontiers in Oncology.

Copyright (C) 2014 Al-Anazi and Al-Jasser. This is an open-access article distributed under the terms of the Creative Commons Attribution License (CC BY). The use, distribution or reproduction in other forums is permitted, provided the original author(s) or licensor are credited and that the original publication in this journal is cited, in accordance with accepted academic practice. No use, distribution or reproduction is permitted which does not comply with these terms. 